\title{
Determination of safe load
}

\author{
S GRACOVETSKY
}

\section{From Concordia University, Montreal, Quebec, Canada}

ABSTRACT The biomechanical criteria adopted by the National Institute for Occupational Safety and Health are shown to be based on considerations that are not consistent with current experi- $\overrightarrow{0}$ mental data and theoretical principles. It is therefore suggested that these standards ought to be replaced, and a rationale for doing so is proposed.

A basic task executed by a manual worker consists of lifting a moderate load, carrying it some distance, then putting it down. The worker may expose his spine to a level of stress that could result in spinal injury, depending on the technique used to lift and carry the load. This paper focuses on this task which is encountered in a typical working environment and illustrates the principles on which the effects of this task on the lumbar spine may be calculated and the risks to the individual assessed.

This problem is not new, and there are many engineering analyses of such simple tasks. From these analyses a "safe level" of load was determined and became widely adopted in the workplace. ${ }^{1}$ Today, it is clear that these safe loads, the type of load which may be lifted and carried without exposing the spine to a predetermined, harmful level of stress, failed to protect the workers' spines. The increasing number of back problems among employees, coupled with the rising costs of repairing their backs, has prompted several governments to set up task forces to investigate the reasons for the apparent failure of the "safe load" to protect the workers' spines.

This paper reviews the biomechanical hypotheses currently accepted by the National Institute of Occupational Safety and Health, and shows that these hypotheses are no longer consistent with available experimental and theoretical evidence, and must therefore be reconsidered. We propose a new approach to determine the level of safe load.

\section{Biomechanical criteria adopted by NIOSH}

The biomechanical criteria adopted by the NIOSH ${ }^{1}$ depend on three physiological parameters: (1) intradiscal pressure, (2) compressive force at L5, and (3) intra-abdominal pressure. We must examine their relevance in the determination of safe loads.

Accepted 22 April 1985
INTRADISCAL PRESSURE

At the turn of the century, anatomists were debating $\vec{N}$ the function of the gelatinous semifluid nucleus of the intervertebral cartilage. They advanced the hypothesis that the nucleus was, by virtue of its water content, an incompressible hydrostatic body. When motion $\mathbb{\Phi}$ occurred, the nucleus acted as a pivot on which the $\frac{0}{2}$ vertebras rocked. With flexion and extension the nucleus was thought to move backwards and forwards. When disc extrusion was found to be the cause $\vec{\varphi}$ of sciatica it was a small step to explain that a digco nucleus could be extruded like a watermelon seedo from between the fingers.

It was soon shown that compression of the disc did not result in nuclear extrusion. Virgin compared the compression strength of joints with and without $\stackrel{\circ}{\Phi}$ nuclei and found little difference. ${ }^{2}$ This was recently $\stackrel{\varrho}{\Rightarrow}$ confirmed by Seyed, ${ }^{3}$ who modelled the disc as a을 hydraulic pressure vessel and showed analytically that the end plate ruptured when the axial load reached $220 \mathrm{~kg}$, which may be compared with the experimentally determined resistance of the joint to destruction (300-700 $\mathrm{kg}^{4}$ ). More importantly, Seyed 3 showed that when the end plate begins to fail, the annular fibres are stretched by less than $4 \%$, which may be compared with their ability to withstand $20 \%$ O stretching.

Therefore, the concept of intradiscal pressure as a음 load bearing mechanism for moderate or heavy lifts is $>$ not supported by experimental evidence and computations. We propose that the annulus is the main N load transmission structure, ${ }^{6}$ and because the annulus ${ }^{\text {or }}$ is made of viscoelastic collagen fibres, the load trans- $N$ mission capacities of the disc are time dependent. Therefore, the amount of load that may be safely lifted depends on time. For example, heavy loads musto be executed at speed because the amount of load that $\bar{\varnothing}$ the annulus can transmit decreases with time. To com- $\stackrel{0}{?}$ pensate for this response the nucleus pressure must 7 
(a)

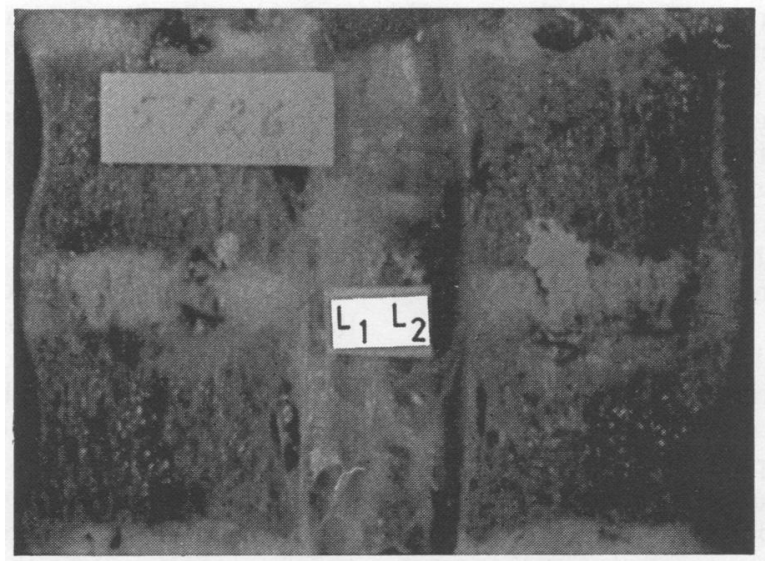

End plates of $L_{1}, L_{2}$ have cracked and nucleus (white dye) has been injected into vertebral body. Note that annulus is intact.

(b)

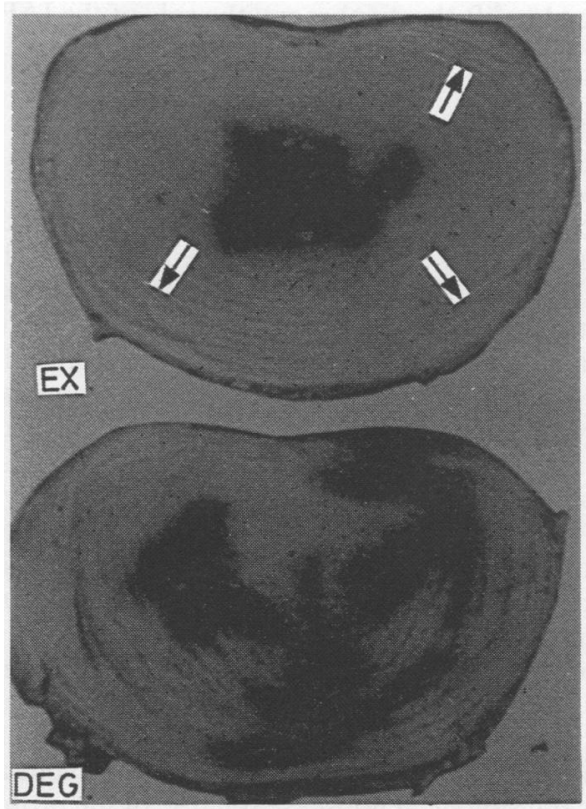

o disc has been subjected in laboratory to torque overload. Note aration of annular layer. Compare with bottom disc which is a iical observation. Notice pronounced layer separation, nucleus has ;rated through damaged layers towards outside of annulus. Such $\because$ is improperly called degenerated. In fact, it is a repeated injury, l further repeated injury would have caused nucleus to escape into al.

1 (a) Compression injury and (b) torsion injury. rise. If the task is not aborted the pressure may rupture the end plate.

When the load to be transmitted is small, there are no theoretical or experimental objections to having the intradiscal pressure rise to transmit the load across the disc. Even then, we note that most tasks are achieved in a second or so, and therefore there is the theoretical possibility that the annulus is, nevertheless, the main load transmission component of the disc.

\section{COMPRESSIVE FORCE}

Keeping in line with the belief that axial compression at the joint and intradiscal pressure were important considerations in explaining lumbar joint injury, Bartelink undertook to develop a mathematical sagittal plane model of the lumbar spine during flexion extension. His model expresses that the external load and body weight is balanced by the action of the erectores with the help of the internal abdominal pressure, pushing the diaphragm upwards. ${ }^{78}$ With this representation the only force applied to the L5-S1 intervertebral joint is compression, and we expect confirmation from pathology, but this is not the case, as is shown by the series of experiments discussed above. In 1969 the spinal mechanism was shown to fail either by excessive compressive load or excessive torsional load. ${ }^{9}$ These modes of failure correspond to the two common pathological sequences seen in postmortem material:

Sequence 1-A central damage to the disc with fracture of varying magnitudes of the end plate is followed 
by an ingrowth of vascular granulation tissue through the fracture into the disc nucleus which results in the gradual destruction of the avascular nucleus and inner annulus. In the early stages the facet joints are not greatly affected. Later, with continued loss of volume of the nucleus, the disc loses its vertical height and the facet joints subluxate. Arthritic changes appear but are rarely severe. The outer annulus survives and is gradually pushed out from between the end plates (fig 1).

Sequence 2-Peripheral damage to both the disc and the facet joints occurring simultaneously is observed. The annulus is avulsed from the end plate and its laminae become separated. The central portion of the disc and the end plate remain intact. At a later stage, the annulus develops radial fissures whereas the nucleus remains relatively untouched. The changes in the facet joints are severe and the intervertebral joint may become unstable (fig 1).

These types of damage may be reproduced in the laboratory by subjecting a joint to excessive compression (sequence 1) or torsion (sequence 2). Hence, we shall refer to them respectively as compression and torsion injuries. In any surgical series we can expect at least $65 \%$ of the cases to be derived from torsional injury and $35 \%$ from compression injury. ${ }^{11}{ }^{12} \mathrm{~A}$ third pathological sequence has not yet been identified, although close to 6000 discs have so far been examined. ${ }^{10} 11$

The compression injury, essentially a fracture of cancellous bone, runs a relatively benign course, and is expected to heal in ten days or so. The torsional injury, on the other hand, is basically a rupture of ligamentous tissue, the annulus, and includes the associated damage to the facet. One facet will be over compressed whereas the other facet's ligaments will have been damaged when the forces prized it open. Ligamentous injuries do not heal quickly. Only $60 \%$ of normal strength is attained after six weeks, and $80 \%$ after six months under best laboratory controlled conditions. Furthermore, this injury may result in instability, which adds another dimension to successful treatment. ${ }^{13}$ Clearly, the costs of this injury may be expected to exceed by far those of the compression type.

Because of the tremendous differences in the nature of these two injuries and their associated costs, any statistical analysis that does not distinguish between these two injuries is bound to be meaningless.

INTERNAL ABDOMINAL PRESSURE

In his model Bartelink initially assigned to the erectores the responsibility for balancing the moment created by the external load and the body weight. According to this model, for a lifted weight of $100 \mathrm{~kg}$ the necessary muscular pull is about $600 \mathrm{~kg}$.
Unfortunately, the maximum muscular output has been determined to be about $250 \mathrm{~kg} .{ }^{14} 15$ (M Hasue et $a l, \mathrm{~T}$ McNeill et al, papers presented at the International Society for the Study of the Lumbar Spine, Goteborg, Sweden, May 1979). Quite clearly, except for small loads, the erectores cannot deliver the required force to balance the external load and the body weight.

This may be appreciated when the cross section of the hip extensor is compared with the erectores. ${ }^{16}$ Intuitively, it is impossible for the forces generated by is the large hip extensors to be "funnelled" through the $\overrightarrow{0}$ erectores. Realising that another supporting member was necessary, Bartelink subsequently proposed that the internal abdominal pressure (IAP) would rise, exerting an upward pressure on the diaphragm, thereby supplementing the activity of the erectores. This in itself is a reasonable proposal and apparently it was accepted without some elementary verifications. Calculations, however, have shown that the required IAP could exceed the arterial blood pressure by ten times, and would halt the flow of blood in the legs. ${ }^{17}$ Such a large calculated pressure has never been observed. Hence, the rise in the IAP cannot be due to the need to lift the diaphragm; the role of the IAP has been described elsewhere. ${ }^{141819}$

\section{Evaluation of a safe load}

\section{RELATING TASK TO INJURY}

To reduce the likelihood of injury, the tasks and the postures that lead to injury must be identified. This is done at present by asking the patient to describe the circumstances leading to his back problem. The task, thus identified, is then analysed. It is impossible, however, to determine which tasks lead to compression injury or torsion injury, or both, since both compression and torsion injuries can give rise to the same symptoms: they both cause back pain and they can both cause back pain and sciatica with or without neurological involvement. Therefore, it is impossible to diagnose a compression or torsion injury from the symptoms.

Current statistical analyses relate symptoms to tasks, and not injury to task, by lumping together compression and torsion injuries into an ill defined "lumbago." Such a mathematically convenient average is physiological nonsense because of the profound differences that characterise these injuries. For example, since torsional injury is by far the most damaging, $N$ it is desirable to reduce the exposure of the spine to N torsion, even if the necessary task modifications would lead to an increase in the risk of compression injury. But first this requires the ability to relate the task to the injury.

The identification of the mode of injury in the 
recently injured is clinically simple. ${ }^{11}$ In the case of a compression failure the patient will have a painful response to increased compression induced by attempting to curl up from the supine position. A torsional failure is detected by a painful response to torsion of the spine in one direction or the other.

We must now examine the types of spinal motion that lead to excessive compression or torsion, or both.

\section{MECHANISM OF THE SPINE}

An ideal mathematical model of the spine should represent all pertinent measurable parameters of spinal function in the performance of a given task. These parameters include the EMG pattern of any muscle, the geometric changes at each intervertebral joint, disc pressure, and the internal abdominal pressure. In addition to satisfying these physiological requirements, the ideal model must also perform within the constraints imposed by the strength of biølogical materials.

We have proposed a model of the spine which calculates these parameters and which has been validated by experiments. ${ }^{141920}$ In addition, other parameters, as yet not measured in vivo, are also calculated. These include ligament tensions and the location of the instantaneous centre of motion, which may be measured in the future.

SUMMARY OF MODEL OF SAGITTAL PLANE MOTION: A WEIGHT LIFT

The basic assumption of this mathematical exercise was that a given task would be performed with a minimum of energy expenditure. As a corollary of this we decided that the task must be performed in such a way that the stress (here the net compressive force acting at the joint, divided by the disc area) is equal and as low as possible at each intervertebral joint.

We analysed the sagittal plane motion of a weight lift in our first study. ${ }^{14}$ The muscular power necessary to execute the lift was derived from the action of the hip extensors. This power must be transmitted to the hands, and the transfer is achieved through the use of both the trunk muscles and the posterior ligamentous system. Hence at each intervertebral joint, the moment generated by muscles and ligaments must equal the moment due to body weight plus the moment due to the weight to be lifted.

The amount of force exerted by any muscle was treated as an unknown, but the direction of this force and its distance from the centre of motion at any joint is known from the coordinates of its attachment points, as determined by anatomical considerations. ${ }^{1621}$ The distribution of moments between muscles and ligament forces was determined by a computer optimisation technique using stress minimisation and equalisation as the objective function.
Several authors have argued that the choice of objective functions in similar optimisation procedures was not critical because "the muscles have the same line of action."1522 These authors did not realise the importance of the ligamentous system and the role of the abdominal muscles in the function of the lumbodorsal fascia, let alone the fact that basic anatomical considerations preclude such mathematically convenient simplifications.

For example, using the compression at L3, as suggested by some, ${ }^{1522}$ leads to the conclusion that it will be advantageous for the spinous process at L5 to be longer than L4, itself longer than L3. Anatomical examinations indicate the opposite. Not surprisingly, by following the same superficial approach it was stated that "whatever the resistance properties of the spine motion segments, the trunk muscles have the capacity to completely overwhelm them. ${ }^{23}{ }^{24} \mathrm{We}$ are not aware of the existence of any vertebrate species that can injure its spine by contracting its trunk muscles. Hence, we must seriously question these simplistic representations.

Ligaments are passive structures, and as such can transmit a force only when stretched. To stretch a ligament, the muscles must modify the spinal geometry in the appropriate way. As a rule of thumb, the muscles must be considered as "gas guzzlers" and, to execute any tasks with minimum energy consumption or minimum stress in all joints, the musculoskeletal system will always attempt to use its ligaments first, and fire a muscle as a last resort. This is mathematically possible because the ligaments (intraspinous, supraspinous, and thoracodorsal fascia) are superficial to the muscles and therefore have better leverage. Thus the use of ligaments has the advantage of lowering the stress at the joint and at the same time minimising energy expenditure. ${ }^{19}$

An illustration of the ligament contribution is given by the action of the abdominals: I oblique and $T$ abdominis are attached to the lateral margin of the thoracodorsal fascia. Contraction of these muscles in the presence of a low abdominal pressure provides a supplementary posterior longitudinal ligament tension that is under direct muscular control. 61819

For weights above $52 \mathrm{~kg}$, the individual must perform the lift at speed because the ligaments are made of viscoelastic fibres that derive additional structural strength and stiffness when strained at speed. When the lift is not executed at speed, the benefit of the ligamentous contribution is lost and the erectores must balance the load. Under these conditions, we calculated that the maximum static load that can be lifted is about $52 \mathrm{~kg}$ - that is, about a quarter of that achievable when the lift is performed at speed. This is in agreement with Chaffin and Park. ${ }^{25}$ We can also calculate other parameters of interest such as the 
not fully accounted for by muscles and ligaments can be easily supported by the facets.

Also, the wide range of spinal motions is commonly believed to induce a wide range of loading configurations of the spine. Hence the vast array of laboratory experiments in which the intervertebral joint is subjected to all types of forces. We have strong reservations regarding this belief, because if this were indeed true the resulting injuries would be equally complex. As indicated above, they are not.

We therefore suggest that the total resultant action of the musculoskeletal system maintains a particular configuration for spinal loading - that is, a compressive force perpendicular to the bisector of the joint, a shear force in the plane of the bisector and absorbed by the facets, and a torque with an axis that coincides with the resultant compression vector. It is not yet clear to us how this conjecture could be proved experimentally.

Subject slowly lifts (3 seconds) a small weight while bilateral EMG activity of sacrospinalis, multifidus, IO, and rectus abdominis is monitored by superficial electrodes. Processing of data is indicated below.

Data from a healthy subject lifting a $5 \mathrm{~kg}$ weight from the floor

(1) EMG of erectores spinae versus time. Signal band filtered and amplified between 10 and $300 \mathrm{~Hz}$. Electrode located at level of $\mathrm{L} 3$ and at $5 \mathrm{~cm}$ to right of spinous process.

(2) Processed EMG versus time. Numbers superimposed indicate instant at which photographs were taken. These permit measurement of the flexion angle alpha.

(3) Processed EMG versus alpha. Compare this curve with calculated muscle moment in (4) below.

(4) Simulated lift. Calculated muscle moment is similar to (3) above, suggesting that ligament contribution is indeed responsible for rapid decrease of erectores activity when flexing forward.

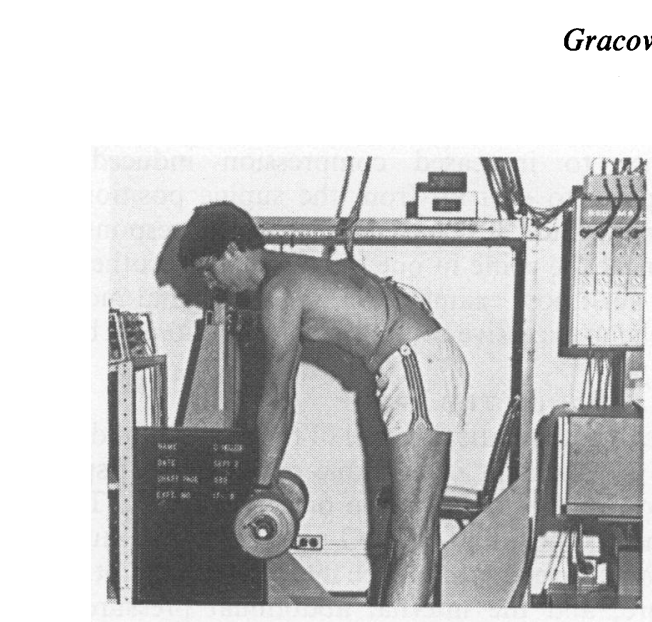

Erectores spinae right

$y \stackrel{\text { w }}{c}$

(1)

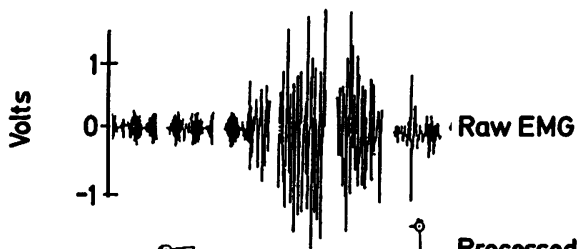

(2)

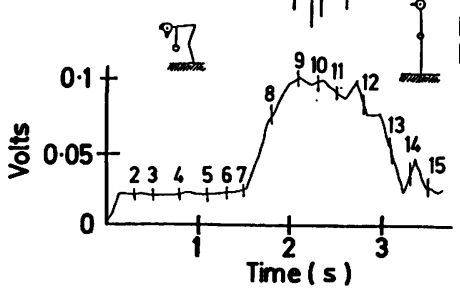

EMG

(3)
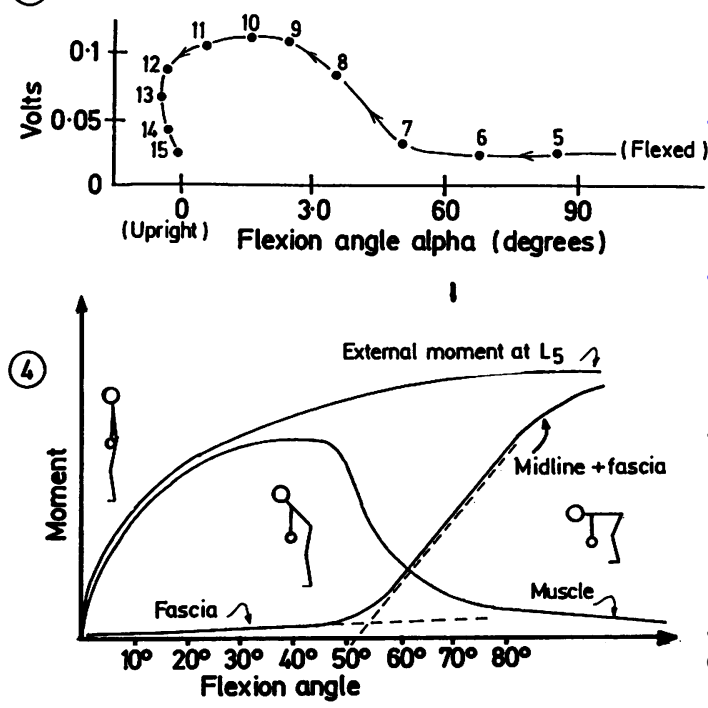

Fig 2 
Importance of flexion

From a normal upright posture, when the pelvic position is maintained, the spine must be flexed forward to about $40^{\circ}$ before the midline ligament becomes tight. It is not necessary, however, to bend forwards to tighten the midline ligament because this may be accomplished by rotating the pelvis backwards by extension at the hips. This permits a person to remain virtually in the upright posture while tightening the midline ligament system and lowering compression stress while remaining upright. Also, a person may then lift a weight by flexing the spine and bending the knees with the lowest possible compression stress in the spine.

The importance of the flexion of the spine while in the erect posture is omitted in the so called "back lift" recommended by Andersson and Troup. ${ }^{20}{ }^{26}$ By insisting that the lordosis be maintained throughout the lift, the mechanism is seriously disabled because the midline ligament is prevented from contributing to the reduction in stress. The lift must then be accomplished by the erectores at a cost of an increase in compressive stress by at least $25 \%$. This technique is, unfortunately, taught by some back schools. ${ }^{27}$

In the range of spinal motion where the midline ligament is slack posterior ligament tension is available through the thoracodorsal fascia. ${ }^{19}$ This ligamentous sheet has roughly the same mechanical advantage as the midline ligament but it is under muscular control throughout the entire range of spinal motion. This system can contribute a moment as large as the erectores.

The teaching of lifting methods must insist on the proper reduction of lordosis and the proper use of the abdominals. Given a choice, the load must be brought as close to the spine as possible to reduce the required external moment. But this must not be done at the expense of the spinal mechanism.

\section{General comments}

(1) A truly safe load in the compression mode has, to date, eluded investigators. At present the standards adopted by the National Institute for Occupational Safety and Health are based on a relatively low proportion of the capabilities of a healthy individual. This leads to the question: can a spine, continuously subjected to a fraction of its capacity, manage to retain its strength?

(2) Our choice of the basic hypothesis of stress minimisation and equalisation is not as arbitrary as it may at first appear. This fundamental hypothesis has permitted us to calculate structure. Our model requires that the length of the $L 5$ spinous process be shorter than that of L4, because otherwise the efficiency of the thoracodorsal fascia would be impaired. Furthermore, because each layer of annulus must support the same stress, we calculated ${ }^{28}$ the length and angle of the fibres in the annular layer, which corresponds closely to those reported. ${ }^{13}$

(3) Our technique is by no means limited to the lumbar spine. We have used it to calculate the maximum acceleration force supportable by the human cervical spine. Our calculated value was $45 \mathrm{~g},{ }^{29}$ corresponding to an in vivo measurement of $47 \mathrm{gg}{ }^{30}$

(4) Because the individual must perform a sagittal plane task according to the fundamental requirement that stress be equalised and minimised, we believe that the pattern of physiological response is independent of structural variation within a wide range, although this remains to be proved. Thus it may be argued that whereas structural lordosis may vary among individuals, the pattern of geometrical change remains the same and the EMG patterns, for example, follow accordingly. The system, however, is changed by injury and the organism must find an alternate method of minimising and equalising the stress, though at a new higher level. This may also be calculated (fig 3).
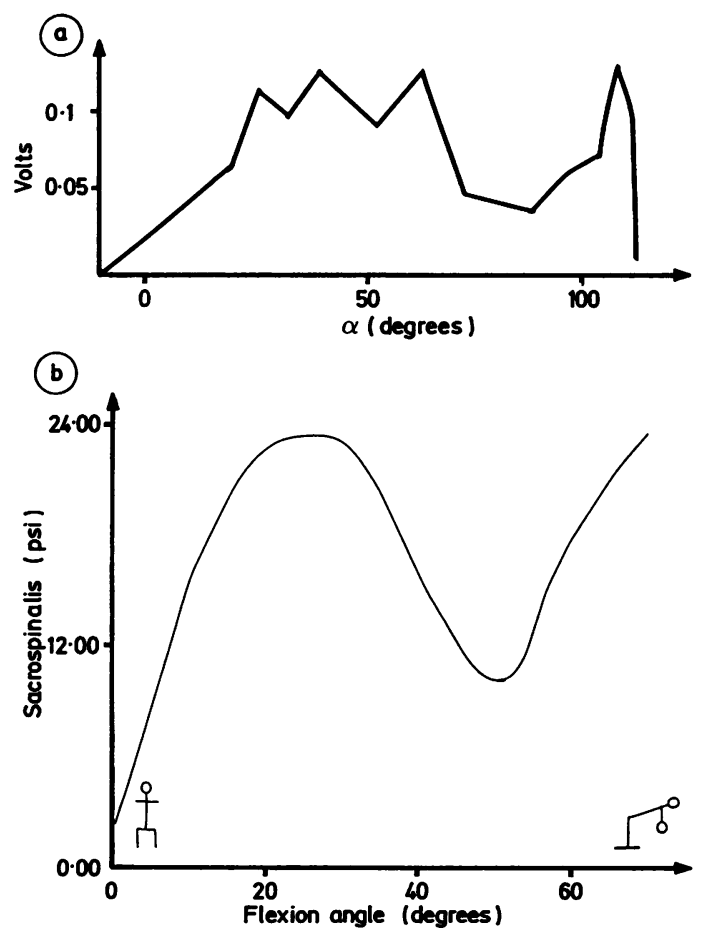

Fig 3 (a) Measured erectores response of a subject with a stiff spine (torsional injury to L4) according to technique described in fig 2. (b) Calculated erectores response of $a$ subject with a stiff spine. A stiff spine is mathematically described by introducing constraits to midline ligament activity because they do not stretch as much as it would if spine were free to flex. Compare with (a). 
OFF SAGITTAL PLANE MOTION: GAIT

At first glance it would appear necessary to reconstruct a three dimensional model to analyse the motion of the spine off the sagittal plane. The paramount importance of the annular fibres of the intervertebral joint suggests that it does not matter how these annular fibres are stretched. Whether by motion in or out of the sagittal plane, it is the reactions of the annulus and the facets that are important. If the equation of the disc could be written and the energy transfers through the disc could be known then the motion of the disc would be derived by expressing that the stress is minimised and equalised for all fibres in the disc, for all discs. The spinal motion could then be reconstructed.

To illustrate the importance of energy transfers in the determination of this behaviour, we propose to examine the requirements of locomotion from the energy point of view and derive the spinal motion.

As early as 1903, Lovett realised that a flexible rod already bent in one plane cannot be bent in another plane without twisting. ${ }^{31} \mathrm{He}$ proceeded to make a fairly complete experimental analysis of the response of the spine when subjected to lateral bending and concluded that an induced axial torque was always present (fig 4).

As the spine is flexed laterally, in addition to the axial rotation, the intervertebral joints are forced to flex forwards, thereby reducing the lordosis. This coupled motion has been described by Farfan $^{9}$ and studied in detail by Panjabi ${ }^{32}$ (M Panjabi, personal communication 1982) on intervertebral joints, and by Pope $^{33}$ on whole cadavers. Thus when an alternating lateral bend is induced in the lumbar spine, the pelvis and shoulders rotate in opposite directions whereas the spine flexes and extends in a predictable manner.

\section{Description of walking}

We will interpret a typical set of data on human gait to show that the proposed theory is consistent with current experimental evidence. It must be appreciated that, by necessity, the experimental data have been taken from different sources, since no one has measured simultaneously all necessary parameters on a single individual. Therefore the calculations derived from these data are perhaps more qualitative than quantitative.

We will use the data collected by Cappozzo ${ }^{234}$ and Thurston. ${ }^{2435}$ After simplification, their measurements may be combined, as in fig 5 . One walking cycle may now be described. As the left leg advances and the right leg is in extension, contraction of the lateral flexors forces the spine to flex to the left, as viewed from the back. The left facet engages and the spine flexes in the sagittal plane as it bends to the left. Spinal flexion must not be confused with forward bending. The spine flexes whenever the lordosis is $\overline{\bar{z}}$ reduced. This effect is evident when the left heel strikes 3 (LHS) and the hip extensors' moment rotates the pel-? vis backwards (fig 5a).

The intervertebral joint is axially loaded ands? torqued to the left by the combined effect of the lateralo flexion and the gravitational field acting on the trunk. 흠 Under such conditions, the coupled motion of the spine generates an axial torque forcing the pelvis to@ rotate clockwise, as seen from above.

It is apparent from fig 5 that the axial torque $\operatorname{Tax}_{\vec{D}}$ is zero at toe off and maximum at heel strike. This. axial torque is the total torque through the L3 level. ${ }^{23} \overrightarrow{\vec{\omega}}$ At toe off, Tax is the sum of at least two equal ando oposite torques:

Passive torque - the torque due to the resistance of the $=$ joint when rotated axially. ${ }^{9}$

Active torque - the torque due to the coupled motioniv of the spine as described above.

Control of pelvic rotation is achieved by control of the induced axial torque-that is, both the lateral bend of the spine and its degree of lordosis. ${ }^{36}$ This $^{-}$ strongly suggests that the pelvis is driven by the spine $\overparen{D}$ and not, as commonly believed, by the legs. This motion is more apparent when the size of the legs is reduced, such as "walking" on the knees.

\section{Problem of torque transmission}

Our description of locomotion requires the spine transmit a large amount of torque, and this is indegd the case. The detailed contribution of the various muscular groups to the torque strength of the intervertebral joint is not known. Nevertheless, because $\frac{\Omega}{\mathbb{\Phi}}$ the maximum static torque strength of the joint is $\overrightarrow{\vec{F}}$ about $20 \mathrm{Nm}^{9}$ and because no vertebrate can destroy을 its joint by contracting its trunk musculature, regardless of what is implied by some, ${ }^{22}$ we conclude that the maximum direct muscular torque on the joint must be less than $20 \mathrm{Nm}$.

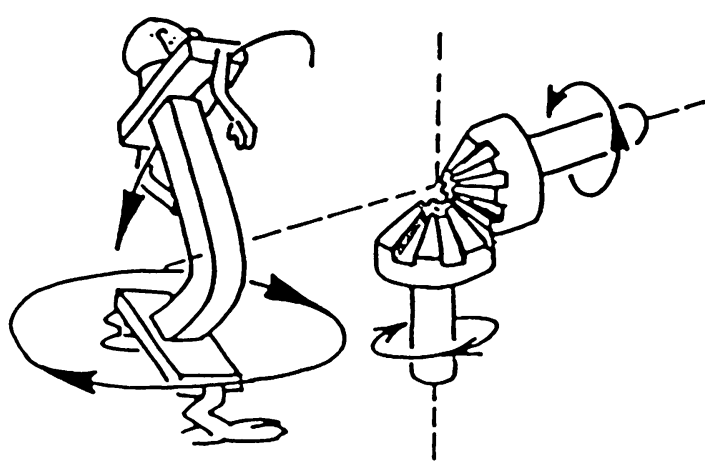

Fig 4 A lateral bend to left produces an axial torque which will force pelvis to rotate clockwise (as viewed from above). 


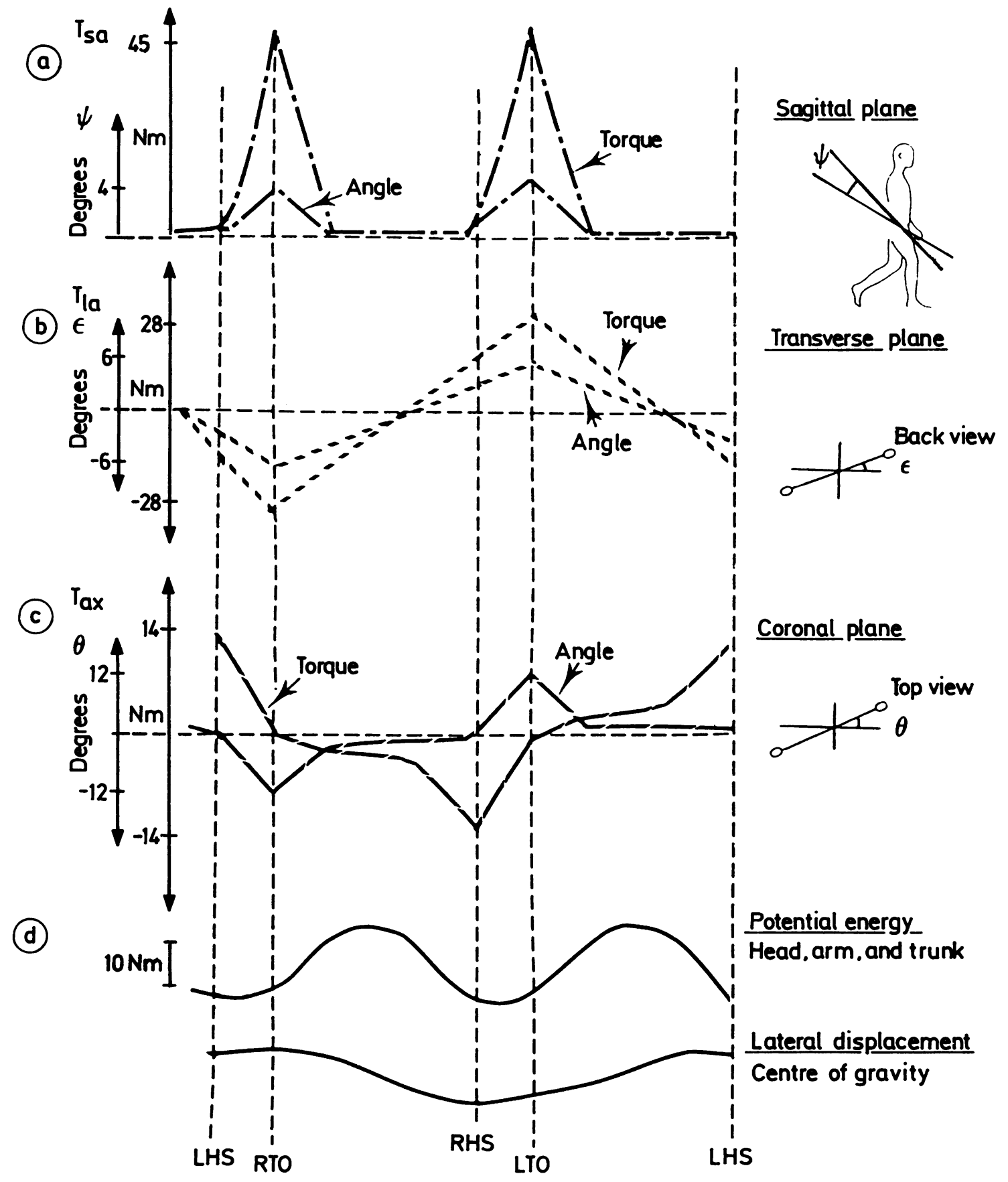

Fig 5 Angular displacement of pelvis with corresponding total torque through L4 in sagittal, transverse, and coronal planes. (Redrawn from Cappozzo ${ }^{23} 34$ and Thurston. ${ }^{24}{ }^{35}$ ) Note correlation between torque and angular displacement in sagittal and transverse planes. Note zero crossover of torque in coronal plane at toe off. 
From the experimental data from Farfan's study ${ }^{10}$ we may calculate that for a walking speed for $2 \mathrm{~m} / \mathrm{s}$ the maximum torque strength of the spine is in the range of 104 to $140 \mathrm{Nm}$. This is considerable.

The resistance of the facets to a static torsional load approximately equals that of the entire annulus. ${ }^{9}$ From this observation, we conclude that the annulus itself must be designed to survive the regular alternating torque generated by the walking motion. In a recent paper we have shown that the orientation of the annular collagen fibres is optimum for the torque strength of the joint. ${ }^{28}$ We suggest that the familiar " $\mathrm{X}$ " pattern may be assigned to the transmission of torsion required for locomotion.

Unfortunately, the fibre orientation of the annulus ${ }^{13}$ has traditionally been viewed and modelled as a system designed to retain a pressurised nucleus, ${ }^{35}$ although the resulting calculations have indicated that the joint would fail at about one quarter of its known ultimate value. It is tempting to visualise the " $X$ " patterns as fulfilling both compression and torsional functions, but it must be remembered that the vertebral body will fail in compression long before the annulus. The data available to date suggest that the structure of the annulus is primarily determined by the requirements of torque transmission.

Nevertheless, we believe that the annulus must somehow fulfil both functions with equal elegance. We suggest that the currently available data on the behaviour of the disc in compression is suspect because both experiments and calculations using this type of loading shows that the end plate ruptures at about a quarter of the ultimate. ${ }^{3}$ This implies that competition weight lifters would routinely crack their end plates whenever they accomplished a lift. There must therefore be something fundamentally wrong with the type of load distribution used in these laboratory experiments, and we suggest they are not representative of in vivo loading patterns.

Our model permits lifts of $200 \mathrm{~kg}$ without exceeding the strength of biological material. ${ }^{6}$ This is achieved with a particular load distribution different from the one adopted by others. ${ }^{3}$ It is not known whether our calculated load distribution is true in vivo. We suggest that it would be reasonable to repeat compression tests using this loading pattern and predict that the joint will exhibit a higher resistance to compression.

The counter rotating masses of arms and legs, besides neutralising angular momentum, instil the capacity for the spine and its ligaments to store kinetic energy released when the motion of the masses is reversed. This energy becomes available for the initiation of the next cycle. We have here, in fact, an oscillator resonating in the gravitational field.

The energy which is stored in the twisted spine may be estimated by approximating the torque curves given by Cappozzo, ${ }^{23}$ and the pelvis angle variations $\overline{\bar{a}}$ given by Thurston ${ }^{2435}$ by sinusoidal functions. Under these conditions up to 70 joules may be stored $\stackrel{0}{?}$ and retrieved at each walking cycle. This is consid- $\vec{F}$ erable because it is of the same order of magnitude of $\stackrel{?}{+}$ the energy variation of the centre of gravity in the gravitational field.

We suggest, therefore, that the problem of energy $\frac{\bar{c}}{\vec{c}}$ transfer within the body may be resolved by consid- $\stackrel{\square}{\square}$ ering that each limb gives to or takes from the spinal engine what it needs.

\section{Control of lordosis}

Lovett noticed that the amount of induced axial $\stackrel{\sigma}{\sigma}$ torque depended critically on the spinal lordosis. ${ }^{31}$ If $\underset{\Phi}{\Phi}$ our interpretation proves to be correct then it is this 3 torque which drives the pelvis. Hence, the control of $\frac{\vec{\omega}}{\omega}$ spinal lordosis appears to be essential in the funda- $i$ mentals of bipedal gait.

Our previous investigations ${ }^{1928}$ have shown that $\stackrel{\sim}{\circ}$ both the deep part of the erectores and the psoas have 을 the potential to control the lateral bend and the lordo- $\rightarrow$ sis, and therefore the induced torque capable of driv- $T^{\top}$ ing the pelvis. Psoas has the additional advantage of $\frac{0}{2}$ being better orientated and its points of attachment are better located than the erectores for that specific $₹$ function. Its activity has been known for some time, ${ }^{39} \vec{\oplus}$ but its functions as a lordosis controller in the loc $\odot$ \% motion process has never been proposed. It wouf therefore be tempting to speculate that the primang function of psoas is the control of lordosis for both flexion-extension and locomotion.

\section{Discussion}

The spinal system needs to be impact loaded, and requires that there be a vertical displacement of the centre of gravity. This is especially true for running when the energy requirements are much greater. $\stackrel{\mathbb{\Phi}}{\mathbb{D}}$ Because the required kinetic energy is obtained from $\exists$ the "free fall" of the centre of gravity, locomotion on rough terrain is not greatly affected.

Like sagittal plane motion, this description neatly explains the known EMG patterns of all the trunk musculature including the abdominals, psoas, the 의 erectores, the hip extensors, and the shoulder girdle $>$ muscles $^{40}$ (fig 6). It illustrates the interaction of the musculoskeletal system with the gravitational field. N

The time dependent feature of biological material is $\mathrm{N}$ a fundamental element in the design of machines $N$ which locomote. An engineer is taught to handle materials, such as steel, which work best when used ing a continuous manner. Perhaps this training is responsible for the long held belief that locomotion is $\frac{\Gamma}{\mathbb{D}}$ best achieved when the centre of gravity is following? a path as linear as possible or that the human machine 7 
Leg acceleration

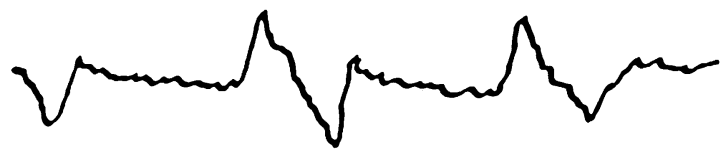

Hip position

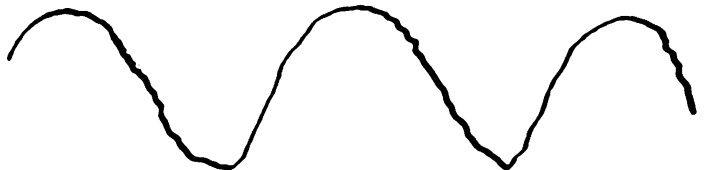

$G$ maximus

similar to left psoas

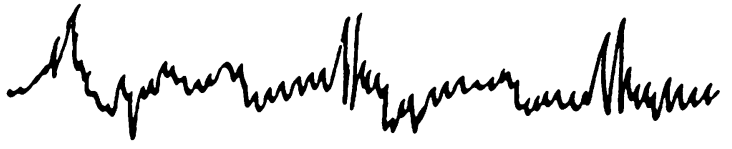

Quad lumborum
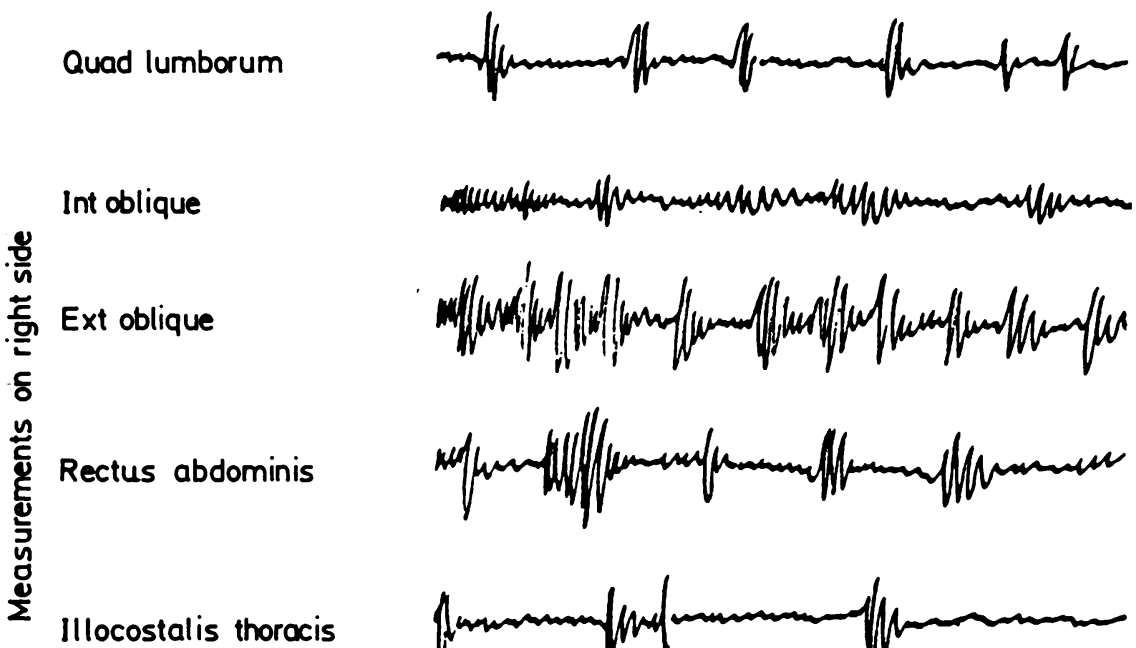

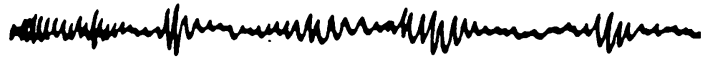
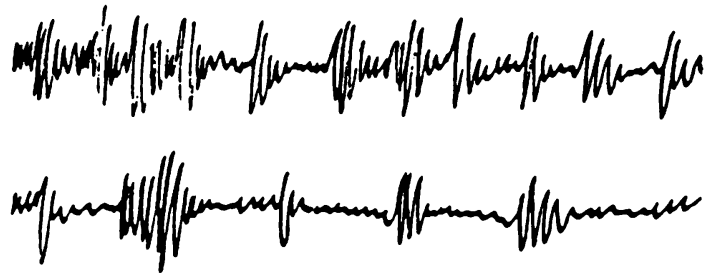

Longissimus thoracis

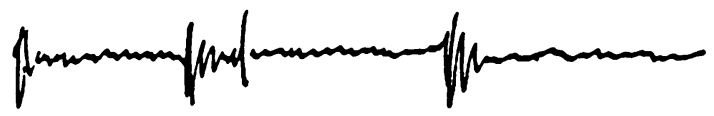

Illocostalis lumborum
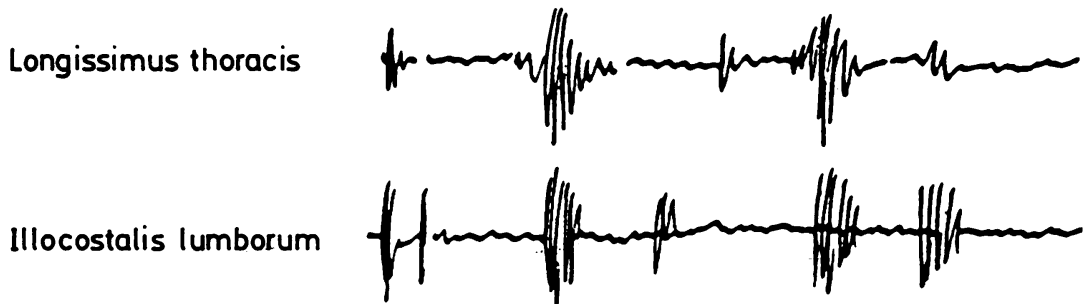

Multifidus

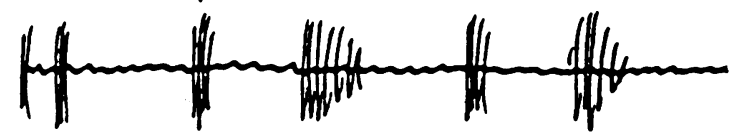

Rotatores
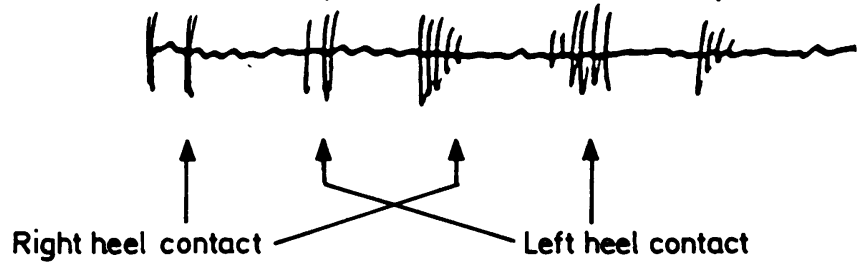

Fig 6 EMG of subjects walking (modified from Waters and Morris ${ }^{37}$ and Liberson ${ }^{38}$ ). 


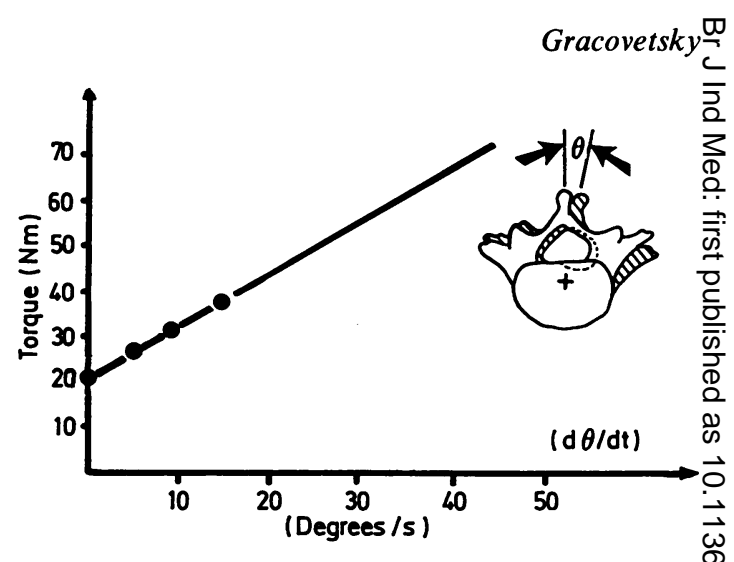

must run smoothly. We recognise here the tendency of some to pad their shoes to dampen what is perceived to be harmful shocks to the spine.

This argument is illustrated in fig 7 which shows the considerable effect of the rate of loading on the torque strength of the intervertebral joint. ${ }^{41}$ Notice that the maximum torque strength in quasistatic mode

$$
\left(\frac{\mathrm{d} \theta}{\mathrm{dt}}=\theta\right)
$$

is about $20 \mathrm{Nm}$. When this value is reported on a remodelled plot of the data collected by Cappozzo (fig 8a) we see that the parabola is intersected at a velocity of $2.57 \mathrm{~m} / \mathrm{s}^{23} 34$ Cappozzo has also measured the vertical force through L3 versus horizontal velocity (fig $8 \mathrm{~b}$ ) and it is remarkable that this vertical force is zero at $2.62 \mathrm{~m} / \mathrm{s}$. We call this the critical speed.

The similarity of the two velocities may be a coincidence due to the limited available data, and further experiments should be completed before concluding that the velocity at which the runner leaves the ground is a measure of the static torque strength of the spine. Beyond this critical speed, as the spine winds and unwinds, resonating in the gravitational field, the energy exchanged between the stretched viscoelastic ligaments and the gravitational field increases. The corresponding vertical displacement of the trunk's centre of gravity is so large that the runner must be airborne.

The importance of the spine as the engine driving the pelvis is more evident as the runner "flies" through the air. It must be noted, however, that there is no essential difference in spinal function between walking and running. The power delivered to the pelvis depends on the rate and amount of lateral flexion associated with the lordosis. There is only so much lateral flexion which the trunk musculature can perform, and, although adequate for slow walking, it is not for running. Hence the term "lateral flexors" of the spine must be enlarged to include all muscles that directly or indirectly flex the spine laterally.

We suggest that the hip extensors take over the role of the trunk musculature as spine flexors in the following way:

(1) The hip extensors' power propels the body through the air as the runner becomes airborne. The energy delivered by the hip extensors is now stored in potential form in the gravitational field. This potential energy is converted into kinetic energy as the runner descends towards the ground.

(2) At heel strike, this energy is recovered by the trunk by the sudden increase in acceleration applied to the trunk mass, forcing it downwards and sideways, thereby forcing the spine to flex. From this point on, the coupled motion of the spine induces an axial torque to drive the pelvis (fig 9).

Fig 7 Torque strength increase versus rate of axial rotation
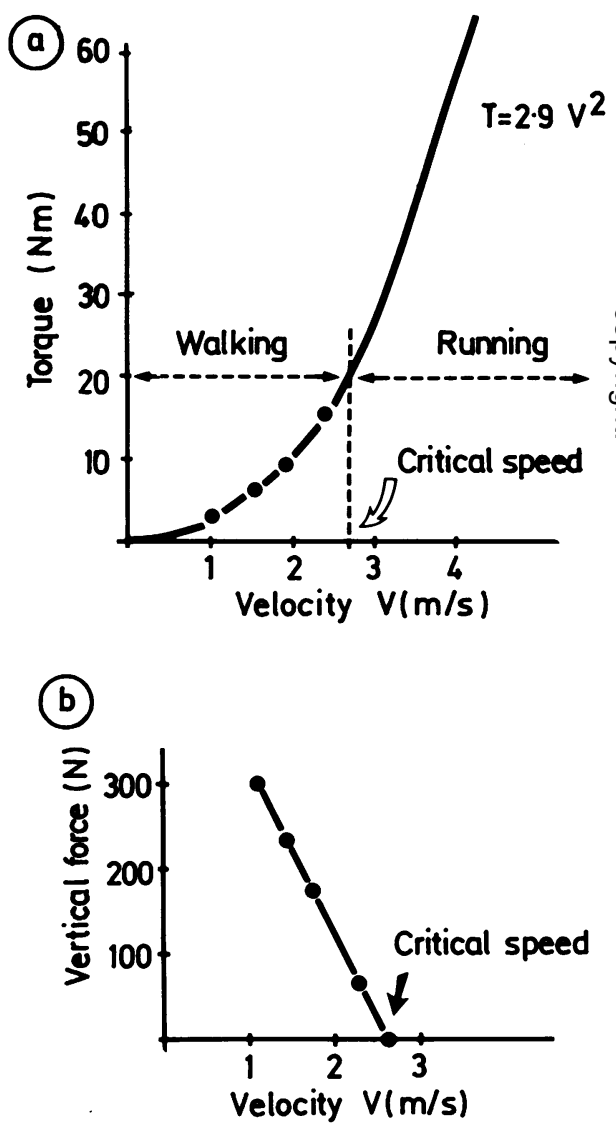

Fig 8 (a) Torque transmitted through $L$ level versus horizontal velocity. $(b)$ These plotted datd were extracted ${ }_{0}$ from measurements of Cappozzo. ${ }^{23}$ Four data points define $\frac{\widetilde{\Phi}}{\mathrm{C}}$ a straight line which intersects horizontal axis for $V=2.6 \%$ $\mathrm{m} / \mathrm{s}$. Compared with (a) axial torque is $20 \mathrm{Nm}$. 
This phenomenon, or change in strategy from walking to running, is fundamentally identical to the switch over that occurs during sagittal weight lifting. When lifting small loads, the weight lifter can use his erectores alone. As the weight to be lifted increases, the erectores cannot deliver the necessary force to balance the external load and body weight. The power of the hip extensors must now be transmitted directly to the upper extremities by the posterior ligamentous system, thereby bypassing the erectores.

Having separately described the mechanism of the spine for weight lifting and walking, we propose to examine the combination of these two basic motions which occur when a worker lifts a weight and carries it at a distance.

\section{Determination of safe load}

Once the load has been lifted and brought close to the body, the worker must now transport it to its destination. When the load is held for a few seconds, the pressure of the disc increases; ${ }^{42}$ this results in the elongation of the annular fibres. The walking process requires the cyclic torque generated by the spinal engine to drive the pelvis. Thus the annular fibres are also subjected to a periodic elongation owing to the need of transmitting torque to the pelvis at each step. The total fibre elongation is therefore due to both axial compression and torque acting on the joint. That is:

$$
\frac{\Delta \mathrm{L}}{\mathrm{L}}=\frac{\Delta \mathrm{L}}{\mathrm{L}} \text { (torsion) }+\frac{\Delta \mathrm{L}}{\mathrm{L}} \text { (compression) }
$$

in which:

$\mathrm{L}$ is the length of fibre at rest,

$\Delta \mathrm{L}$ (torsion) is the elongation of the fibre resulting from an axial torque applied to the joint, and

$\Delta \mathrm{L}$ (compression) is the elongation of the fibre resulting from an axial compression.

The relation between $\Delta \mathrm{L}$ and the angle of axial rotation has been calculated, ${ }^{28}$ the relation between axial torque and axial rotation may be deduced from experiments. ${ }^{9}$

The relation between the torque transmitted by the joint, the rate of rotation $\mathrm{d} \theta / \mathrm{dt}$, and the maximum angle of rotation of the joint $\theta_{\max }$ may be derived from $^{43}$ and shown in fig $8 b$.

$\mathrm{T}_{\mathrm{j}}=\mathrm{A}^{*} \mathrm{~d} \theta / \mathrm{dt}+\mathrm{B}^{*} \boldsymbol{\theta}$

where $T_{j}$ is the torque supported by the joint, $\theta$ is the angle of axial rotation, and $A$ and $B$ are coefficients that are functions of the axial load.

When the spine is loaded we expect the coefficients $A$ and $B$ to change. The combination of $\theta$ and $\mathrm{d} \theta / \mathrm{dt}$ chosen by the body to execute the walk could be obtained by a minimisation procedure expressing that this optimum combination results in minimum torque stress at the joint. Thus we would obtain a value for $\theta$ which, if less than $2^{\circ}$, could be considered safe. ${ }^{9}$ The precise value depends on the data $A$ and $B$ which so far had been determined experimentally, only in the case where torque is applied without axial load $(\mathrm{A}=$ 1.2 and $B=5 \cdot 7)$. This ideal laboratory condition is not representative of true walking and hence $A$ and $B$ are not known in the general case.

We will, however, proceed by taking the value of $\mathrm{A}$ $=1.2$ and $B=5.7$, keeping in mind the above remarks. Once the fibre arrangement is known, ${ }^{28}$ the relation between $\Delta \mathrm{L}$ (compression) and the axial compression is determined by using the experimental data given by Seyed. ${ }^{3}$ Because the collagen fibres are made of viscoelastic material, the total elongation $\Delta \mathrm{L}$ is a function of time, compression, and torsion.

The results of such calculations are given in fig 10 . Such calculations are probably of qualitative value only; true experimental data giving the response of the joint to periodic axial rotations with axial load is not available.

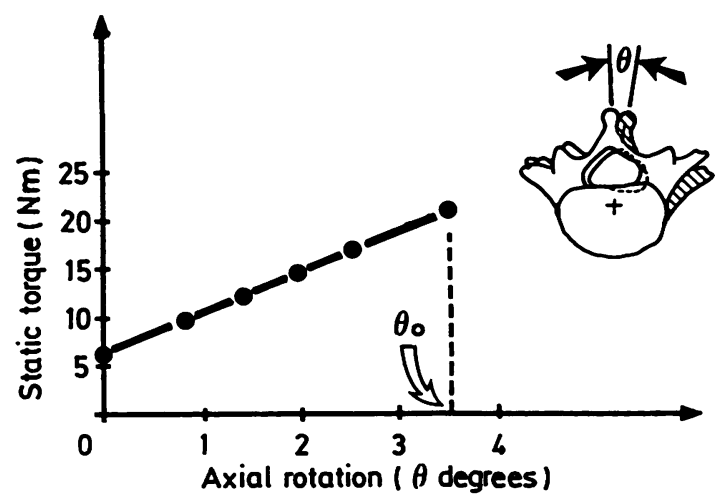

Fig 9 Experimental determination of axial rotation with applied axial torque.

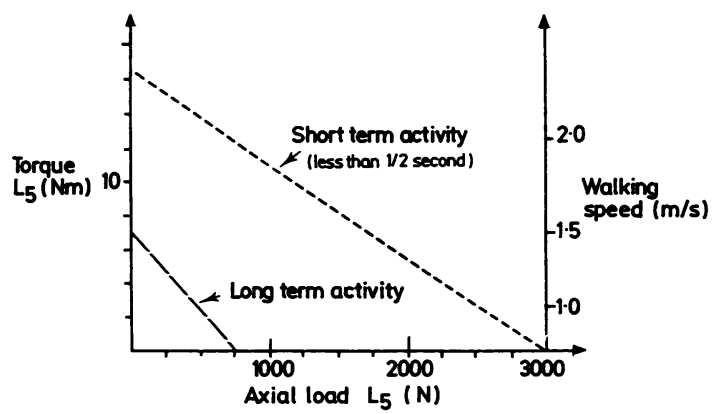

Fig 10 Safe load limits. Safe limit is calculated from the requirement that relative elongation of ligament fibres is less than 5\%. Maximum permissible values are shown for two cases and range of activity for each case is represented by area below dotted and dashed lines. 


\section{Long distance walking}

As the spine flexes, the midline ligament is activated and is able to transmit forces. This, in turn, permits the erectores to reduce their activity by an amount equal to what the midline takes. In fig 2 it is seen that this transition zone occurs, when the external load is zero, for an angle of about $30-40^{\circ}$ of forward flexion. When the external load increases, the transition zone is shifted towards the origin.

This muscle/ligament switching phenomenon represents a mechanism for transferring the load from ligament to muscle thereby allowing either one to rest in an alternating manner. The pelvic rotation at each step is indeed modifying the spinal lordosis and is therefore controlling the switching of muscle to ligament.

Hence, the typical forward attitude of a hiker carrying a backpack. By permitting small oscillations at each step, he alternately loads and rests his muscles and midline ligaments.

\section{Walking with a heavy load}

To walk with a heavy load the spine must transmit enough torque to rotate the pelvis. Additional axial load stiffens the intervertebral joint ${ }^{9}$ (M Panjabi, personal communication) so that, for a given transmitted torque, the angle of axial rotation is reduced, thereby reducing the amplitude of pelvic oscillation, thereby shortening the stride.

To reduce the stress in the joint the spine must be flexed to use its posterior ligaments. The reduction in lordosis reduces the ability of the spine to bend laterally, and impedes the development of torque in the lumbar spine. Walking becomes difficult as this torque is reduced.

Thus if the individual must walk with an axial load, he must maintain some lordosis. The amount of lordosis required is controlled by the speed at which he is required to walk.

For any given walking speed the impact of heel strike provides the energy for the spinal engine to drive the pelvis. Additional axial load increases the energy at impact and therefore increases the torque at the intervertebral joint. This may be corrected by reducing the lordosis, thereby diminishing the induced torque, and consequently the oscillation of the pelvis and the length of the stride. One recognises the almost running, short stepped gait of a porter carrying a heavy load.

An excellent example of walking with an increased axial load is the man with a wheelbarrow. The load is applied axially to the spine by having the handles of the barrow firmly held to the side of the body. After bending to lift the wheelbarrow the man must restore his lordosis so that he can develop the correct amount of torque for walking. The worker should have a reduced walking stride and he should not be in full $\overline{\bar{z}}$ lordosis otherwise both torque and compression stress would increase.

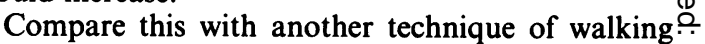
with an axial load often seen in the Far East, where the $\vec{F}$ load is divided into two baskets suspended on a pole $\stackrel{0}{0}$ resting on the shoulders. In this technique again the vertical alignment of the load with the spine is $\frac{\bar{c}}{\frac{D}{D}}$ assured. In addition, there is no restraint on the rota- $\frac{\vec{\sigma}}{\vec{\alpha}}$ tion of either the pelvis or the shoulders. This confers $\propto$

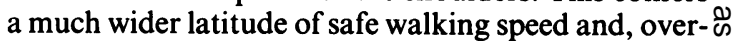
all, a better chance for the reduction of stress.

\section{Conclusion}

We have shown that the NIOSH biomechanical crite- $\mathbb{D}$ ria for safe load are not in line with current experimental and theoretical data. Safe load is a function of $\hat{\omega}$ the weight to be lifted, its bulkiness, time, and the ? walking speed at which it must be transported.

\section{References}

${ }^{1}$ National Institute for Occupational Safety and Health. Work prac- $\frac{\mathbb{D}}{O}$ tices guide for manual lifting. Cincinnati, Ohio: DHHS (NIOSH) 1981. (Publication No 81-122.)

${ }^{2}$ Virgin W. Experimental investigation into the physical properties of the intervertebral disc. J Bone Joint Surg 1951;33B:607-11.

${ }^{3}$ Seyed A, Shrivastavi S, Ahmed A. Stress analysis of the lumbar disc $\infty$ body unit in compression; a three-dimensional nonlinear finfe? element study. Spine 1984;9:120-33.

${ }^{4}$ Markolf KL, Morris J. The structural components of the intevertebral disc; a study of their contribution to the ability of $\bar{T}$ disc to withstand compressive forces. $J$ Bone Joint Surg $\overline{\bar{O}}$ 1974:56A:675-7.

${ }^{5}$ Sonnerup L. Semi-experimental stress analysis of the human intervertebral disc in compression. Experimental Mechanics 1972;12:142-7.

${ }^{6}$ Gracovetsky S, Farfan H. The optimum spine. Spine (in press).

${ }^{7}$ Bartelink DL. The role of the abdominal pressure in relieving the pressure on the lumbar intervertebral disc. J Bone Joint Surg 1957;39B:718-25.

${ }^{8}$ Morris GM, Lucas DB, Bresler B. Role of the trunk in the stability of the spine. J Bone Joint Surg 1961;43A:327-51.

${ }^{9}$ Farfan HF. Mechanical disorders of the lumbar spine. Philadelphia: Lea \& Febiger, 1973.

${ }^{10}$ Farfan H, Cossette J, Robertson G, Wells R, Krauss H. The effects of torsion on the intervertebral joint: the role of torsion in the 0 production of disc degeneration. J Bone Joint Surg 1970; 3 52A:468-97.

${ }^{11}$ Farfan F. The use of mechanical etiology to determine the efficacy $\stackrel{\circ}{\supset}$ of active intervention in single joint lumbar intervertebral joint $>$ problems: surgery and chemonucleolysis compared, a prospec-음 tive study. Spine 1985;10:350-8.

${ }^{12}$ Kirkaldy WW, Farfan H. The present status of spinal fusion. Clin $\mathrm{N}$ Orthop 1981;158:198-214.

${ }^{13}$ Galante JO. Tensile properties of the human lumbar annulus $N$ fibrosus. Acta Orthop Scand 1967;suppl No 100.

${ }^{14}$ Gracovetsky S, Farfan H, Lamy C. The mechanism of the lumbar $\omega$ spine. Spine 1981;6:249-62.

${ }^{15}$ Schultz AB, Andersson G, Ortengren $R$, Haderspeck $K$ Nachemson A. Loads on the lumbar spine: validation of a bio- $\frac{C}{(\Phi)}$ mechanical analysis by measurements of intradiscal pressures $\mathscr{S}$ and myoelectric signals. J Bone Joint Surg 1982;64A:713-20. 
${ }^{16}$ Hollinshead W. Anatomy for surgeons: the back and limbs. Vol 3. New York: Harper and Row, 1969.

${ }^{17}$ Farfan H, Gracovetsky S, Helleur C. The role of mathematical models in the assessment of tasks in the workplace. In: International series of biomechanics: Biomechanics $I X-B$. Proceedings of the 9 th International Congress of Biomechanics. Champaign, IL: Human Kinetics Publishers, Inc, 1985:38-43.

${ }^{18} \mathrm{Gracovetsky} \mathrm{S}$. The function of the spine inflexion-extension. $J$ Biomed Engin (in press).

${ }^{19}$ Gracovetsky S, Farfan H, Helleur C. The abdominal mechanism. Spine 1985;10:317-24.

${ }^{20}$ Ortengren R, Andersson G, Nachemson A. Studies of relationships between lumbar disc pressure, myoelectric back muscle activity, and intra-abdominal pressure. Spine 1979;6:98-103.

${ }^{21}$ McClintic JR. Basic anatomy and physiology of the human body. New York: John Wiley and Sons, 1975.

${ }^{22}$ Schultz A. Biomechanics of the spine. Proceedings of conference on low back pain and industrial and social disablement. London: Back Pain Association, 1982.

${ }^{23}$ Cappozzo $A$. The forces and couples in the human trunk during level walking. J Biomech 1983;16:265-77.

${ }^{24}$ Thurston AJ, Whittle MW, Stokes IAF. Spinal and pelvic movement during walking: a new method of study. Eng Med 1981;10:219-22.

${ }^{25}$ Chaffin DB, Park KS. Longitudinal study of low back pain as associated with occupational weight lifting factors. Am Ind $\mathrm{Hyg}$ $J$ 1973;34:513-25.

${ }^{26}$ Troup JDG. Dynamic factors in the analysis of stoop and crouch lifting methods: a methodological approach to the development of safe materials handling standards. Orthop Clin North Am 1977;8:201-9.

${ }^{27}$ Apts DW, Blankership KL. Back facts. Ashland, Kentucky: The American Back School, FRP Inc publishers (1505 Carter Ave, suite 301, 41105-1193), 1981.

${ }^{28}$ Farfan H, Gracovetsky S. The nature of instability. Spine 1984;4:714-9.
${ }^{29}$ Helleur C, Gracovetsky S, Farfan H. Tolerance of the human cervical spine to high acceleration: a modelling approach. Aviat Space Environ Med 1984;55:903-9.

${ }^{30}$ Stapp JP, Major MC. Human exposure to linear deceleration. The forward facing position and the development of crash harnesses. US Armed Forces, 1951. (AF technical report No 5915-part II.)

${ }^{31}$ Lovett RW. A contribution to the study of the mechanics of the spine. Am J Anat 1903;2:457-62.

${ }^{32}$ White A, Panjabi M. Clinical biomechanics of the spine. Philadelphia: JB Lippincott Co, 1978.

${ }^{33}$ Pope MW, Wilder D, Matteri R, Frymoyer J. Experimental measurements of vertebral motions under load. Orthop Clin North Am 1977;8:155-67.

${ }^{34}$ Cappozzo A, Figura F, Marchetti M. The interplay of muscular and external forces in human ambulation. J Biomech 1976; 9:35-43.

${ }^{35}$ Thurston AJ, Harris JD. Normal kinematics of the lumbar spine and pelvis. Spine 1983;8:199-205.

${ }^{36} \mathrm{Krauss} \mathrm{H}$. Effect of lordosis on the stress in the lumbar spine. Clin Orthop 1976;117:56-8.

${ }^{37}$ Waters RL, Morris JM. Electrical activity of muscles of the trunk during walking. J Anat 1972;111:191-9.

${ }^{38}$ Liberson WT. Biomechanics of gait: a method of study. Arch Phys Med Rehabil 1965;46:37-48.

${ }^{39}$ Nachemson A. Electromyographic studies on the vertebral portion of the psoas muscle. Acta Orthop Scand 1966;37:177-90.

${ }^{40}$ Basmadjian J. Muscles alive-their function revealed by electromyography. Baltimore: Williams and Wilkins Company, 1979.

${ }^{41}$ Krauss H, Farfan H, Jones T. Stress analysis of human intervertebral disc. Proceeding of 25th Annual Conference on Engineering in Medicine and Biology. 1972:242.

${ }^{42}$ Nachemson A. Disc pressure measurements. Spine: symposium on low back pain in industry 1981;6:93-7.

${ }^{43}$ Gracovetsky $S$. An hypothesis for the role of the spine in human locomotion: a challenge to current theory. J Biomed Engin 1985;7:205-16. 